\title{
BMJ Open Robot-assisted radical cystectomy with intracorporeal urinary diversion versus open radical cystectomy (iROC): protocol for a randomised controlled trial with internal feasibility study
}

\author{
James W F Catto, ${ }^{1,2}$ Pramit Khetrapal, ${ }^{2}$ Gareth Ambler, ${ }^{3}$ Rachael Sarpong, ${ }^{4}$ \\ Muhammad Shamim Khan, ${ }^{5}$ Melanie Tan, ${ }^{4}$ Andrew Feber, ${ }^{2}$ Simon Dixon, ${ }^{6}$ \\ Louise Goodwin, ${ }^{1}$ Norman R Williams, ${ }^{4}$ John McGrath, ${ }^{7}$ Edward Rowe, ${ }^{8}$ \\ Anthony Koupparis, ${ }^{8}$ Chris Brew-Graves, ${ }^{4}$ John D Kelly ${ }^{2}$
}

To cite: Catto JWF, Khetrapal P, Ambler G, et al. Robotassisted radical cystectomy with intracorporeal urinary diversion versus open radical cystectomy (iROC): protocol for a randomised controlled trial with internal feasibility study. BMJ Open 2018;8:e020500. doi:10.1136/ bmjopen-2017-020500

- Prepublication history for this paper is available online To view these files, please visit the journal online (http://dx.doi. org/10.1136/bmjopen-2017020500).

Received 9 November 2017 Revised 17 May 2018 Accepted 7 June 2018
Check for updates

(C) Author(s) (or their employer(s)) 2018. Re-use permitted under CC BY-NC. No commercial re-use. See rights and permissions. Published by BMJ.

For numbered affiliations see end of article.

Correspondence to Dr Pramit Khetrapal; p.khetrapal@ucl.ac.uk

\section{ABSTRACT}

Introduction Bladder cancer $(\mathrm{BC})$ is a common malignancy and one of the most expensive to manage. Radical cystectomy ( $\mathrm{RC}$ ) with pelvic lymphadenectomy is a gold standard treatment for high-risk BC. Reductions in morbidity and mortality from RC may be achieved through robot-assisted RC (RARC). Prospective comparisons between open $\mathrm{RC}(\mathrm{ORC})$ and $\mathrm{RARC}$ have been limited by sample size, use of extracorporeal reconstruction and use of outcomes important for ORC. Conversely, while RARC is gaining in popularity, there is little evidence to suggest it is superior to ORC. We are undertaking a prospective randomised controlled trial (RCT) to compare RARC with intracorporeal reconstruction (iRARC) and ORC using multimodal outcomes to explore qualitative and quantitative recovery after surgery.

Methods and analysis $\mathrm{iROC}$ is a multicentre prospective RCT in English National Health Service (NHS) cancer centres. We will randomise 320 patients undergoing $\mathrm{RC}$ to either iRARC or ORC. Treatment allocation will occur after trial entry and consent. The primary outcome is days alive and out of hospital within the first 90 days from surgery. Secondary outcomes will measure functional recovery (activity trackers, chair-to-stand tests and health related quality of life (HRQOL) questionnaires), morbidity (complications and readmissions), cost-effectiveness (using EuroQol-5 Domain-5 levels (EQ-5D-5L) and unit costs) and surgeon fatigue. Patients will be analysed according to intention to treat. The primary outcome will be transformed and analysed using regression. All statistical assumptions will be investigated. Secondary outcomes will be analysed using appropriate regression methods. An internal feasibility study of the first 30 patients will evaluate recruitment rates, acceptance of randomised treatment choice, compliance outcome collection and to revise our sample size.

Ethics and dissemination The study has ethical approval (REC reference 16/NE/0418). Findings will be made available to patients, clinicians, funders and the NHS through peer-reviewed publications, social media and patient support groups.
Strengths and limitations of this study

- This is the first clinical trial to compare qualitative recovery after open and robotic radical cystectomy.

- The use of intracorporeal reconstruction and enhanced recovery makes this a comparison of the optimal robotic approach against the optimal open surgery.

- Many surgeons are learning robot-assisted radical cystectomy and so a learning curve element may impact the experimental arm.

Trial registration numbers ISRCTN13680280 and NCT03049410.

\section{INTRODUCTION}

Context

Bladder cancer (BC) is a common malignancy and one of the most expensive to manage. ${ }^{2}$ Around $25 \%$ of BCs present with muscle invasion BC (MIBC), and these tumours have an ominous prognosis. ${ }^{3}$ Radical cystectomy (RC), with pelvic lymphadenectomy, represents the gold standard treatment for MIBC and plays a key role in managing high-risk non-muscle invasive cancer and in salvage after radiotherapy. ${ }^{4} \mathrm{RC}$ is a major operation associated with frequent comorbidity. ${ }^{56}$ Reductions in morbidity and mortality from RC have occurred in recent years through refined anaesthesia, improved surgical techniques, enhanced recovery pathways and centralisation of services into high volume centres. ${ }^{7-9}$ Further refinements may be achieved through robot-assisted surgery. 


\section{Current knowledge: robotic and open RC (ORC)}

For most abdominal surgery, minimally invasive approaches are less morbid than open surgery and expedite postoperative recovery without altering cancer cure. ${ }^{10}$ Within minimally invasive techniques, robot-assisted surgery offers improved dexterity, precision and vision, with less surgeon fatigue than traditional laparoscopy. However, there is little or conflicting evidence of benefit from robot-assisted RC (RARC) over the open approach, ${ }^{11-13}$ and there are uncertainties about oncological outcomes with robotic surgery. ${ }^{14}$ This may reflect the complex nature of this procedure (involving surgery to both the urinary and gastrointestinal tracts), limitations of the current evidence, the typical patient cohort (elderly, smoker, pre-existing cardiopulmonary disease) or a true lack of difference. Prospective comparisons between ORC and RARC have been of limited size and design. ${ }^{15-18}$ For example, a three-arm comparison randomised 60 patients to open, laparoscopic or robotic extracorporeal RC. ${ }^{16}$ Each arm had a single surgeon, and the sample size prevented robust comparisons. The largest published study to date found no difference in postoperative outcomes for 118 patients in two arms. ${ }^{15}$ This trial was criticised as the extracorporeal route mitigated potential benefits of RARC, ${ }^{19}$ and there was an unexplained patient preference for ORC (7\% randomised to RARC opted for ORC vs $0 \%$ in other direction). The largest prospective study (307 patients) of ORC versus extracorporeal RARC is yet to report. ${ }^{17}$

\section{Recovery after surgery}

Recovery after laparoscopic and open surgery is mostly compared using length of stay (LOS), operating time and procedure costs. However, LOS is affected by Enhanced Recovery After Surgery (ERAS) pathways, healthcare design and social care facilities and misses postdischarge readmissions. Readmission is common and can be prolonged following RC. ${ }^{20}$ Operating times and procedural costs appear robust, but they can reflect surgeon choice and experience, and billing practice rather than procedural outcomes. ${ }^{21}$ Finally, these measures focus solely on patient-based rather than surgeon-based metrics.

\section{Surgeon fatigue}

The impact of surgeon fatigue on patient care, and the ability of robot assistance to reduce this, is poorly studied and understood. ${ }^{22}{ }^{23}$ Validated measures of workload (eg, SURG-TLX tool ${ }^{24}$ ), mental effort and gaze control can assess surgeon fatigue and be used to compare robotic and open surgery. Previous work has demonstrated that surgeons perform tasks more quickly and accurately with fewer errors on a robotic system. ${ }^{25}$ Self-reported measures of workload and mental effort were significantly lower on a robotic compared with laparoscopic system. Similarly, an objective cardiovascular measure found less mental effort was needed on a robotic platform compared with laparoscopy. ${ }^{26}$ These findings suggest tasks can be performed more proficiently, with less workload and mental effort using a robot. ${ }^{26}$ This may allow surgeons greater cognitive resources for dealing with other demands such as communication, decision making or periods of increased complexity in the operating room. There may also be implications for productivity in the operating room as surgeons appear capable of delivering a higher number of complex procedures per theatre day compared with open or laparoscopic surgery.

\section{Research need}

To determine differences between iRARC and ORC may require a multidimensional approach. With regards to hospital stay, a parameter such as days alive and out of hospital (DAOH) during a predefined perioperative period $^{27}$ (including LOS and readmission) will give a more realistic estimate of recovery time. Post operative complication rates and quality of life (QoL) measures can be more discriminatory between techniques, as they directly reflect postoperative return to normal life and the smoothness of the perioperative period. There are various ways to compare patient experience and QoL. These include hospital outcomes, such as LOS and postoperative complications, and assessment instruments such as EQ-5D-5L, ${ }^{28}$ WHO Disability Assessment Schedule 2.0 (WHODAS-2) $^{29}$ and European Organisation for Research and Treatment of Cancer (EORTC) Quality of Life Questionnaire (QLQ)-Bladder Muscle-invasive 30 questions (BLM30). ${ }^{30} 31$ In addition, functional tools, such as wearable tracking devices (eg, Fitbit) or activity tests can be used to quantitate activity over time. As such, we are undertaking a prospective randomised controlled trial (RCT) to compare recovery, safety and outcomes from ORC and iRARC.

\section{Study aims and outcomes}

We aim to prospectively randomise eligible patients to either iRARC or ORC and measure their recovery in order to compare oncological outcomes, safety and cost-effectiveness and determine the role of both approaches in patient care. Our primary outcome is:

i. To compare the number of $\mathrm{DAOH}$ within 90 days from surgery.

Secondary outcomes will assess recovery, complications, cost-effectiveness and surgeon fatigue across five domains:

\section{Recovery from surgery}

The following tools will measure recovery:

i. Overall functional recovery: self-administered WHODAS-2 (12-point) questionnaire at baseline (preoperative), 1 month, 3 months, 6 months and 12 months after surgery.

ii. Cystectomy specific impact on HRQOL: EORTC QLQ-BLM30 and Quality of life questionnaire cancer 30 questions (QLQ-C30) questionnaires at baseline (preoperative), 1 month, 3 months, 6 months and 12 months after surgery.

iii. Quantified activity levels: steps (total and daily average) taken over consecutive days (measured using a 
wearable tracking device, eg, Fitbit test) at baseline (7 days preoperative), 5 days postoperative, 1 month ( 7 days), 3 months (7 days), 6 months (7 days) and 12 months ( 7 days).

iv. 30 Second chair to stand test: number of times the patient can stand from sitting in a $30 \mathrm{~s}$ interval. This will be counted in the outpatients clinic at baseline (preoperative), 1 month, 3 months, 6 months and 12 months. ${ }^{32} 33$

v. Qualitative and economic analysis: EQ-5D-5L at baseline, 1 month, 3 months, 6 months and 12 months.

\section{Perioperative morbidity}

To assess perioperative morbidity, the following data will be collected:

i. Adverse events recorded using the Clavien-Dindo classification.

ii. Readmission to hospital within 90 days of surgery.

iii. Intraoperative and postoperative blood transfusion rates.

iv. Length of days in critical care.

v. Rate of radiological or surgical intervention.

vi. Readmission to intensive treament unit (ITU).

vii. 30-Day and 90-day mortality rate.

viii. Conversion from iRARC to open ORC.

ix. Conversion from intracorporeal to extracorporeal reconstruction.

\section{Oncological outcomes}

To assess the oncological outcomes from surgery, the following data will be collected:

i. The number of retrieved lymph nodes in the pathological specimen.

ii. Positive margin rate in the pathological specimen.

iii. Port site recurrence free survival.

iv. Location of recurrence/metastases.

v. Overall and cancer-free survival at 12 and 18 months.

\section{Surgeon fatigue}

A substudy will evaluate surgeon fatigue. Specifically, immediately following surgery, for 50 IRARC and 50 ORC cases, the surgeons will be tested using a validated psychometric test called SURG-TLX. ${ }^{34}$ This tool questions mental demand, physical demand, temporal demand, performance and effort and quantifies response using a Likert scale. ${ }^{26}$

\section{Cost-effectiveness}

Cost-effectiveness and quality-adjusted life years (QALYs) of iRARC against ORC will be estimated using resources used, unit costs and EQ-5D-5L (collected at baseline, 1 month, 3 months, 6 months and 12 months).

\section{METHODS AND DESIGN}

\section{Trial design}

iROC is a prospective multicentre RCT in patients undergoing RC for BC. Eligible patients will be consented and randomised 1:1 to iRARC or ORC after multidisciplinary team (MDT) review in National Health Service (NHS) cancer centres. It is not possible to blind patients or staff to the treatment received. The primary outcome will be measured 90 days after RC and secondary outcomes up to 12 months. Patients will include those receiving neoadjuvant chemotherapy (typically gemcitabine and cisplatin) and those having either an ileal conduit or a neobladder reconstruction.

\section{Trial population}

We aim to recruit 320 patients (figure 1), with an internal analysis after the first 30 days. Prior to entry, patients must be accurately staged (eg, cross-sectional imaging (eg, CT) of the abdomen, pelvis and thorax or bone scan if indicated), within 3 months prior to randomisation and judged to be eligible for both treatments. Anaesthetic evaluation will be used in those with borderline fitness. After trial entry, women of childbearing age must be proven to be not pregnant (pregnancy test). Eligible participants will fulfil all the inclusion criteria and none of the exclusion criteria as defined below:

\section{Inclusion criteria}

i. Participants must be over 18 years of age.

ii. Histopathological confirmation of BC (Urothelial Cell Carcinoma (UCC), Squamous Cell Carcinoma (SCC), adenocarcinoma or rare variant).

iii. Carcinoma in situ (CIS) or stage pTa or pT1 or $\geq \mathrm{pT} 2$ or mobile bladder mass on bimanual examination under anaesthesia.

iv. Node status $\leq \mathrm{N} 1$ on imaging criteria or positron emission tomography (PET)-ve outside pelvis.

v. Eastern Cooperative Oncology Group (ECOG) grade 1,2 or 3.

vi. Able to give informed written consent to participate.

Exclusion criteria

i. Unwilling to undergo cystectomy.

ii. Previous abdominal surgery rendering them unsuitable for either iRARC or ORC.

iii. Patients with upper urinary tract disease.

iv. Concomitant disease that would render the patient unsuitable for the trial.

v. Pregnant or lactating females.

vi. Previous radiotherapy for BC.

Eligibility waivers are not permitted.

\section{Sample size}

The primary outcome measure is $\log (90-\mathrm{DAOH})$ within the first 90 days from surgery. This is expected to be approximately normally distributed. For the power calculation, we estimated the characteristics of this measure using information on LOS (given the relatively small proportion of deaths) in the 2015 British Association of Urological Surgeons (BAUS) national dataset. We aim to detect a difference between groups of 0.22 units, assuming a conservative SD of 0.7 units. Using a $5 \%$ significance level and $80 \%$ power, it is calculated that 160 subjects per 


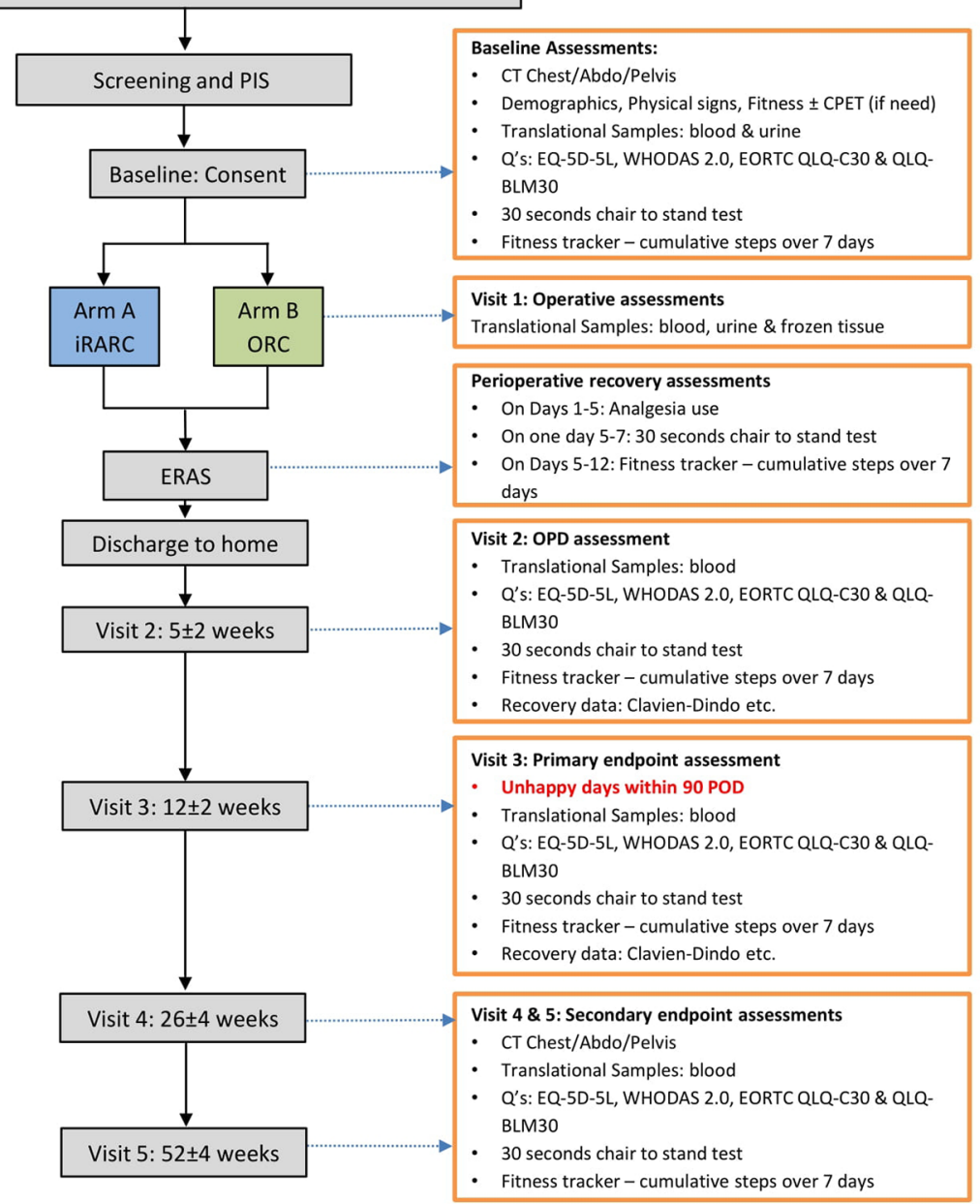

Figure 1 Detailed study schema. CPET, cardiopulmonary exercise testing; iRARC, RARC with intracorporeal reconstruction; OPD, outpatients department; ORC, open RC; PIS, patient information sheet; POD, post-operative day; RARC, robot-assisted $\mathrm{RC}$; RC, radical cystectomy; SMDT, Surgical Multi-disciplinary team.

group, 320 in total, are required for the study. Thus, we aim to recruit 320 patients.

We note that the original primary outcome was $\log (\mathrm{DAOH})$. However, a review of blinded internal pilot data suggested that this outcome would be left skewed and that other sample size assumptions would not hold.

\section{Internal feasibility study}

There is a recognition that surgical RCTs can be hard to recruit to and that patients may not accept their allocated treatment option..$^{35}$ Furthermore, with respect to iROC, little is known about the secondary outcome activity measures in the context of post-RC recovery. As such, we will undertake an internal feasibility study of the first 30 patients to look at rates of recruitment, acceptance of allocated treatment and collection of outcomes. In addition, the statistician will use blinded aggregate data to check the assumptions used to calculate the sample size. If necessary, this will be used to revise our sample size.

\section{Patient and public involvement (PPI)}

Patient and public stakeholders were made aware of the unmet need for research following the NHS England Specialised Services Clinical Commissioning Policy $(16033 / \mathrm{P})$ statement to withdraw RARC based on lack of evidence. Patient feedback on study design was obtained through The Urology Foundation's (Charity no. 1128683) peer review process, which includes PPI representatives, and through Fight Bladder Cancer (Charity no. 1157763). Furthermore, PPI representatives are on the Trial Steering Committee (TSC) for iROC and will have oversight of the management of the research and analysis.

On completion of the study, results will be disseminated to patients through their urologists. The results will be 
published following peer review, and anonymised data will be presented at national and international conferences.

\section{Setting}

Participants will be recruited from NHS cancer centres undertaking both ORC and iRARC. Recruiting centres will be invited by the Trial Management Group (TMG) as having well-developed RARC programmes with sufficient volume to recruit a reasonable number of patients to the trial.

\section{Surgeon and unit accreditation}

Variations in surgical team performance produce wide differences in outcomes from RC. ${ }^{36}$ Surgeons and surgical teams undertaking radical surgery within this study require accreditation from the TMG. Surgeons undertaking iRARC need to have completed more than 30 iRARC (as sole surgeons) and have submitted these data to the BAUS Oncology database. Surgeons will also need to submit an unedited anonymised video of one entire procedure to the trial TMG for review. At the start of recruitment, the TMG will organise surgeon training days to discuss the surgical aspects of iRARC. Accredited surgeons will have undertaken more than 10 RCs per year for the last 2 years as primary surgeon, have a median LOS under 14 days and will have 90-day post-RC mortality rate of less than $5 \%$. Individual surgeon data will act as surrogate measures for the entire surgical team.

\section{Radical cystectomy}

$\mathrm{RC}$ will be performed as is standard of care throughout the NHS. In females, this includes anterior pelvic exenteration (with the uterus, fallopian tubes and a component of the anterior vaginal wall). The urethra will be excised in females choosing an ileal conduit. Oophorectomy is individualised for each patient, as per local practice. In males, RC includes the prostate and seminal vesicles. Nerve sparing to the prostatic neurovascular bundles should be attempted as per typical practice. Exceptions to this surgical plan are acceptable with prior approval from TMG. Pelvic lymphadenectomy should be included in all cases, unless contraindicated clinically. The lymphadenectomy template should include the external iliac, obturator and internal iliac nodes, with a proximal extension to the level of the ureteric crossing of the common iliac vessels. A more extended lymphadenectomy is acceptable. Excised lymphatic tissue should be submitted for histological analysis. Urinary tract reconstruction within this trial is limited to either: (1) ileal conduit or (2) orthotopic neobladder (by whichever design is practised by that unit (eg, Tan $\left.\left.e t a l^{37}\right)\right)$.

\section{Intervention A: iRARC}

Intracorporeal robot assisted radical cystoprostatectomy or anterior exenteration should be conducted as per local practice using an Intuitive da Vinci system. The procedure should include intracorporeal reconstruction using small bowel. Surgeon discretion is allowed for the placement of a pelvic drain, nasogastric tubes, the use of local anaesthesia and port site closure. All specimens should be removed using impermeable collections bags or surgical gloves. Measures to avoid spillage of urine or fluid from the specimens must be taken.

\section{Intervention B: ORC}

ORC should be performed using a midline incision as per local protocols. The procedure should include urinary reconstruction using small bowel. Surgeon discretion is allowed for the placement of a pelvic drain, nasogastric tubes, the use of local anaesthesia and wound closure. Regional anaesthesia using rectus sheath blocks or an epidural is recommended.

\section{Recruitment}

iROC will recruit patients attending NHS cancer centres. All patients who have a diagnosis of $\mathrm{BC}$ in whom the specialist MDT have recommended RC as a treatment option can be approached.

\section{Consent}

Written informed consent will be obtained from each patient prior to study entry and performing baseline trial assessments. A patient information sheet and informed consent form are provided to facilitate this process. The investigator, or their designee, must ensure adequate explanations of the study, that participation is voluntary, and they can withdraw at any time. In consenting to the study, participants are consenting to provide study follow-up and data collection. Consent to provide liquid biopsy (blood, urine) as well as permission to obtain paraffin blocks will be requested. Patient who do not wish to consent for these translational samples remain eligible for the randomised trial. A patient may withdraw from the study at any time without prejudice to his or her subsequent treatment.

\section{Randomisation}

Patients will be randomised, using an online system (https://www.sealedenvelope.com/trials/) on a 1:1 basis to either iRARC or ORC. A computer-generated adaptive minimisation algorithm that incorporates a random element will be used to ensure the treatment groups are balanced (stratified) for:

- Centre.

- Type of urinary diversion (continent diversion/ neobladder or ileal conduit).

Treatment allocation will occur after trial entry and consent.

\section{Data collection}

Trial assessments will be conducted at various time intervals (defined around the date of surgery). Schedule of events is summarised in table 1. Time points:

i. Baseline/preoperative: at the time of consent and trial entry.

ii. Perioperative: operative details collected immediately after surgery. 
Table 1 Timing of events and outcome collection within iROC

\begin{tabular}{|c|c|c|c|c|c|c|c|}
\hline & Baseline & RC & Postoperative & Visit 2 & Visit 3 & Visit 4 & Visit 5 \\
\hline Time & Preoperative & Day 0 & POD 4-7 & $\begin{array}{l}5 \text { weeks } \pm 2 \\
\text { weeks }\end{array}$ & $\begin{array}{l}12 \\
\text { weeks } \pm 2 \\
\text { weeks }\end{array}$ & $\begin{array}{l}24 \\
\text { weeks } \pm 4 \\
\text { weeks }\end{array}$ & $\begin{array}{l}1 \text { year } \pm 4 \\
\text { weeks }\end{array}$ \\
\hline Informed consent and randomisation & $x$ & & & & & & \\
\hline $\begin{array}{l}\text { Demographic data, medical history and } \\
\text { so on }\end{array}$ & $\mathrm{x}$ & & & & & & \\
\hline Physical examination and vital signs & $x$ & & & & & & \\
\hline Fitness for surgery assessment & $x$ & & & & & & \\
\hline 12-lead ECG and CPET testing* & $x$ & & & & & & \\
\hline Haematology and biochemistry & $x$ & & & $x$ & $x$ & $x$ & $x$ \\
\hline Pregnancy test $†$ & $\mathrm{x}$ & & & & & & \\
\hline Translational and research bloods $\ddagger$ & $x$ & & & $x$ & $x$ & $x$ & $x$ \\
\hline Urinalysis & $\mathrm{x}$ & & & & & & \\
\hline Urine collection for research $\ddagger$ & $x$ & & & & & & \\
\hline Chest, abdomen and pelvis imaging§ & $\mathrm{x}$ & & & & & $\mathrm{x}$ & $\mathrm{x}$ \\
\hline Clavien-Dindo assessment & & & $\mathrm{x}$ & $x$ & $x$ & & \\
\hline Adverse events & & $\mathrm{x}$ & $\mathrm{x}$ & $x$ & $\mathrm{x}$ & $\mathrm{x}$ & $\mathrm{x}$ \\
\hline Tumour sample & & $\mathrm{x}$ & & & & & \\
\hline Paraffin embedded tissue & & $x$ & & & & & \\
\hline Survival and treatment data & & & $\mathrm{x}$ & $x$ & $\mathrm{x}$ & $\mathrm{x}$ & $x$ \\
\hline EQ-5D-5L & $x$ & & & $x$ & $\mathrm{x}$ & $\mathrm{x}$ & $\mathrm{x}$ \\
\hline WHODAS 2.0 & $x$ & & & $x$ & $x$ & $x$ & $\mathrm{x}$ \\
\hline EORTC QLQ-BLM30 & $x$ & & & $x$ & $\mathrm{x}$ & $\mathrm{x}$ & $\mathrm{x}$ \\
\hline 30 s chair to stand test & $x$ & & $\mathrm{x}$ & $x$ & $\mathrm{x}$ & $x$ & $x$ \\
\hline Quantified activity levels (steps tracker) & $x$ & & $x$ & $x$ & $x$ & $x$ & $\mathrm{x}$ \\
\hline
\end{tabular}

${ }^{*}$ Cardiopulmonary exercise testing only in participating centres that routinely perform this test.

†Can be urine or blood-based pregnancy test, depending on site's standard of care.

†Home collection kit to be given.

§Imaging schedule is not mandated by the trial. However, if imaging studies have been conducted, these results are collected.

$\mathrm{POD}$, postoperative day; RC, radical cystectomy.

iii. In-hospital postoperative: recovery from surgery, complications and secondary interventions after surgery, activity/mobility at 5 days after RC.

iv. Hospital discharge: time (days) to leaving hospital for home.

v. Within 90 days of surgery: readmission to the hospital, self-completed questionnaires.

vi. Outpatient follow-up: at 5 weeks, 3 months, 6 months and 12 months from the date of surgery questionnaires, fitness tracking data and blood tests.

The time point of interest for measurement of the primary endpoint will be 90 days. Patients will continue to receive routine clinical follow-up (at 4-6 weeks, 3 months, 6 months, 12 months). Subject to additional funding, we also propose a future extension to collect overall and cancer-free survival data at 18 months.

\section{The secondary endpoint measures include}

i. EQ-5D-5L (baseline, 5 weeks, 3 months, 6 months and 12 months): a self-completion tool for patients that is applicable to a wide range of health conditions and treatments. Measured domains include mobility, self-care, usual activities, pain and anxiety or depression. ${ }^{28}$

ii. WHODAS 2.0 (baseline, 5 weeks, 3 months, 6 months and 12 months): a generic assessment instrument for health and disability used across all diseases, including mental, neurological and addictive disorders directly linked at the level of the concepts to the International Classification of Functioning, Disability and Health. WHODAS 2.0 covers six domains of functioning, including cognition, mobility, self-care, getting along, life activities and participation. ${ }^{29}$

iii. EORTC QLQ-BLM30 Version 1 (baseline, 5 weeks, 3 months, 6 months and 12 months): a 30 -item questionnaire for patients with BC (T2, T3, T4a and T4b). This module is designed to be used in conjunction with QLQ-C30 and includes an assessment of urinary symptoms, bowel symptoms, sexual functioning, 
urostomy problems, problems associated with the use of a catheter and body image. ${ }^{30} 31$

iv. Quantified activity levels (baseline, 5 days, 5 weeks , 3 months , 6 months and 12 months). Fitness tracking devices will record steps taken for seven consecutive days at predetermined time points. ${ }^{38}$ The WHO $30 \mathrm{~s}$ sit to stand test will also be administered at these time points. $^{32}$

v. Operative: intraoperative surgical details will be recorded (intraoperative case report form (CRF)). Missing data extracts will be obtained from the BAUS complex cancer database. The following operative measures will be recorded:

- Duration of surgery (total and individually for cystectomy, lymph node dissection (LND), urinary diversion times).

- Grade of operator.

- Training op (yes/no).

- Conversion rate.

- Operative blood loss.

- Operative blood transfusion rate.

- Visceral injury.

- Vascular injury.

- Death.

- Return to theatre.

vi. Postoperative: an inpatient postoperative assessment CRF will be used to record progress. Clavien-Dindo classification of surgical complications will be used to assess for any surgical complications as per normal hospital practice. These scores will be recorded at 30 and 90 days postcystectomy. ${ }^{39} 40$

vii. Oncological outcomes (3 months, 6 months and 12 months): the curative outcomes from the RC will be examined at 3,6 and 12 months to determine local and distant recurrence, metastases, need for palliative treatment and survival (overall and cancer specific). CT scans will be undertaken at 12 months, according to usual practice, and if clinically indicated.

viii. Cost-effectiveness: the study will follow good practice guidelines for economic evaluations undertaken alongside controlled trials. Differences between patients in the two arms will be calculated and cost-effectiveness will be compared against funding thresholds used by the National Institute for Health and Care Excellence (NICE). Costs will be calculated from resources used and unit cost. Key resources are robotic equipment, theatre consumables, theatre time and staffing, length of hospital stay, complications, readmissions and ambulatory care (eg, outpatients, generap practitioner (GP) visits and nurse visits), and these will be prospectively collected within each hospital and in an ambulatory setting. Unit costs (collection prices) will be derived from NHS business managers and NHS Tariffs for each centre contributing 20 or more cases into iROC. We will calculate total costs and QALYs for each patient and estimate the incremental costs and QALYs using a seemingly unrelated regression model with baseline covariates including age and baseline EQ-5D-5L score and missing data imputed using multiple imputation (EQ-5D$5 \mathrm{~L}$ collected at baseline, 5 weeks, 3 months, 6 months and 12 months)). QALYs will be estimated using EQ$5 \mathrm{D}-5 \mathrm{~L}$ values using the official EuroQoL Group tariff and using linear interpolation between time points. Following this analysis, bootstrapping will be used to generate the cost-effectiveness plane and associated cost-effectiveness acceptability curves. Deterministic sensitivity analyses will be undertaken using the EQ5D-3L tariff using a crosswalk algorithm. Undertaking a sensitivity analysis with the $3 \mathrm{~L}$ tariff is required as the $5 \mathrm{~L}$ tariff has yet to be adopted by NICE or accepted more generally by economists/researchers.

\section{Statistical analyses}

A detailed statistical analysis plan will be written before any analysis is undertaken. All analyses and data summaries will be conducted using the groups to which the patients were randomised and patient will be included regardless of whether they received the allocated treatment (intention to treat (ITT)). Statistical tests and CIs will be two sided, and estimates of treatment effects will be presented with $95 \%$ CIs and significance will be considered at the $5 \%$ level. No formal interim analyses are planned.

The flow of study participants will be displayed in a Consolidated Standards of Reporting Trials diagram, and the baseline characteristics of the patients will be summarised by trial arm using appropriate descriptive statistics. The primary analysis will be a comparison of the transformed primary outcome $\mathrm{DAOH}$ using regression to adjust for the minimisation factors. The secondary outcomes will also be analysed using appropriate regression methods. The use of mixed models will be considered for those secondary outcomes measured at several time-points. All statistical assumptions will be investigated. Potential bias due to missing data will be investigated by comparing descriptively the baseline characteristics of the trial participants with complete outcome measurements to those who have missing outcome measurements. The use of regression (to adjust for variables related to missingness) and multiple imputation methods (chained equations) will be considered as sensitivity analyses. Patients will be offered the option of telephonic follow-up if they are unable to attend clinic appointments for follow-up. Additionally, patients wishing to withdraw from the trial will be counselled regarding end of active participation, as this would allow the trial team to collect outcome data for an ITT analysis. For patients who consent into the trial but ultimately do not undergo RC, only survival data will be collected.

\section{Safety}

The number of adverse events and related unexpected serious adverse events (SAEs) will be summarised descriptively by arm, by grade and body system. The proportion of participants experiencing each toxicity will be summarised by maximum NCI CTCAE grade $^{41}$ 
experienced, overall and by arm. RC is a major operation that has a number of recognised complications and a low risk of death (less that 1 in 100). Expected adverse events will be reported as per previous reports ${ }^{56}$ :

- Gastrointestinal (29\%): ileus, small bowel obstruction (SBO), constipation, clostridium difficile colitis, gastrointestinal bleeding, emesis, anastomotic bowel leak and diarrhoea.

- Infectious (25\%): fever of unknown origin (FUO), abscess, urinary tract infection (UTI), sepsis, urosepsis, pyelonephritis, diverticulitis, gastroenteritis and cholecystitis).

- Wound (15\%): wound seroma, wound infection, wound dehiscence and facial dehiscence/evisceration.

- Genitourinary (11\%): renal failure, ureteral obstruction, urinary leak, urinary fistula, urinary retention, parastomal hernia, stomal ischaemia and haematuria.

- Cardia (11\%): arrhythmia, myocardial infarction, hypertension, congestive heart failure, angina and hypotension.

- Pulmonary (9\%): atelectasis, pneumonia, respiratory distress, pneumothorax, and pleural effusion.

- Bleeding (9\%): anaemia requiring transfusion, postoperative bleed other than GI and wound haematoma.

- Thromboembolic (8\%): deep vein thrombosis, pulmonary embolism and superficial phlebitis.

- Neurological (5\%): peripheral neuropathy, cerebrovascular accident transient (CVA)/transient ischaemic attack (TIA), delirium/agitation, vertigo, loss of consciousness and seizure.

Operative/postoperative RC complications will be graded using the Clavien-Dindo classification. ${ }^{39}{ }^{40}$ All SAEs will be recorded in the medical records, the CRF, the sponsor's adverse event (AE) log and an SAE form. The principal investigator (PI) or designated individual will complete an SAE form, and the form will be faxed to surgical and interventional trials unit (SITU) within five working days of becoming aware of the event. The chief or PI will respond to any SAE queries raised by the sponsor as soon as possible. Where the event is unexpected and thought to be related to the procedure, this must be reported by the investigator to SITU, who will then inform the Health Research Authority within 15 days.

\section{Data monitoring}

This study will use an electronic case report form (eCRF), and trial data will be entered into an approved, protected database (https://www.elsevier.com/solutions/macro). Access to the eCRF system will only be provided to staff with relevant authority. Participants will be given a unique subject number and subject identifier. Data will be entered under this identification number onto the central database stored on the servers. The database will be password protected and only accessible to members of the iROC study team and external regulators if requested. The servers are protected by firewalls and are patched and maintained according to best practice. The physical location of the servers is protected by CCTV and security door access. The database software provides a number of features to help maintain data quality, including: maintaining an audit trail, allowing custom validations on all data, allowing users to raise data query requests and search facilities to identify validation failure/missing data. After completion of the study, the database will be retained on the servers of University College London for ongoing analysis of secondary outcomes. The identification, screening and enrolment logs, linking participant identifiable data to the pseudoanonymised subject numbers will either be held in written form in a locked filing cabinet or electronically in password-protected form on hospital computers. After completion of the study, the identification, screening and enrolment logs will be stored securely by the sites for 10 years unless otherwise advised by sponsor.

\section{Trial funding, organisation and administration}

The trial was developed by the iROC TMG and funded by The Urological Foundation and Champniss Charitable Trust. Intuitive Surgical has contributed disposable surgical equipment to the iRARC arm but has had no role in trial design and will have no role in trial implementation, analysis, interpretation or writing any reports. The trial is sponsored by University College London and registered with ISRCTN (13680280) and ClinicalTrials.gov (NCT03049410) on 10 February 2017. Members of the trial team are Good Clinical Practice (or equivalent) trained. A Data Monitoring Committee (DMC) will monitor patient safety and the rate of recruitment of subjects in the study. They will meet at least once a year while the trial is ongoing for routine review of safety data and trial progression. They have the power to call additional meetings and review data at any point in the trial should they wish to do so. The DMC may report their findings to the TSC. The TSC is an independent committee consisting of relevant, experienced clinicians and researchers. The TSC will ensure the study is conforming to governance requirements as set out by the trial sponsor. The TSC will meet at least once a year. The sponsor may also arrange an independent trial monitor to review the study data.

\section{DISCUSSION}

Since its introduction, robot-assisted surgery has rapidly gained popularity despite a lack of evidence of its superiority over other approaches. For example, in England, most NHS Radical Prostatectomies are performed using robot assistance (https://www.baus.org.uk/patients/ surgical_outcomes/radical_prostatectomy/default. aspx). With regards to RC, in 2016, $40 \%$ of the 1279 RCs submitted to the BAUS Complex Operation database (which covers $91 \%$ of cases in England and Wales using healthcare quality improvement partnership (HQIP) data) involved robotic surgery. This compares to $31 \%$ 
in 2015 and $14.5 \%$ in 2014. While single surgeon series demonstrate favourable outcomes from robotic surgery, ${ }^{42}$ prospective clinical trials and population surveys have found minimal benefit that may not justify the additional cost. ${ }^{43-45}$ This has left many healthcare funders in a difficult position. For example, a NHS England Specialised Services Clinical Commissioning Policy $(16033 / \mathrm{P})$ stated it would no longer routinely commission RARC, despite the trend to increase RARC within the NHS.

The popularity of robotic surgery among patients, surgeons and hospitals suggests that observed marginal benefits may be more important than the data suggest or that other benefits have not been demonstrated. With this in mind, we developed iROC to test a fully intracorporeal approach against ORC to focus on the quality of patient recovery and to test surgeon fatigue. $\mathrm{RC}$ may be an ideal procedure for robotic assistance given its surgical complexity, typical comorbid patient cohort and potential for economic savings from reduced morbidity and faster hospital discharge. RC is a two-stage procedure involving: (1) removal of the bladder and (2) urinary tract reconstruction. Following robot-assisted bladder removal, many surgeons undertake reconstruction through an open incision (so called extracorporeal). This is less technically challenging than robotic intracorporeal reconstruction but leads to increased cost (open and robotic instruments needed) and leaves the patient with both robotic and open incisions. To date, prospective comparisons of open and robotic RC have been limited by the use of an extracorporeal reconstruction. To overcome this limitation, we have mandated intracorporeal reconstruction in iROC.

Current data and RCTs have also used primary outcomes that matter most in open surgery (eg, transfusion rate, LOS and pathology). To overcome this, we have additionally focused on qualitative recovery. However, little is known of these qualitative outcomes after RC (either open or robotic) and patient compliance with the collection of these measures. We have planned an internal pilot phase ( 30 patients) to review secondary outcome collection and may adapt sample size or outcome collection following this.

\section{ETHICS AND DISSEMINATION}

The results of the study will be published in peer-reviewed publications and will be presented at relevant national and international conferences. We will work with our patient panel to develop lay reports to disseminate research findings to patient groups and the clinical teams at participating sites.

\section{Availability of data}

SITU, the CTU will control the final trial dataset, and any requests for access will be reviewed by the TMG and TSC and subject to existing contractual arrangements with the funders. The protocol, sample case report forms and participant information are available on a case by case basis as agreed by the TMG, on request to the corresponding author.

\section{TRIAL STATUS}

The trial opened to recruitment in March 2017 using protocol version 1.0 (dated November 2016) and is due to close in February 2020 or after accrual and the last patient visit is completed. The protocol was amended to version 2.0 in May 2017 to account for additional inclusion and exclusion criteria, and updated surgeon accreditation criteria. Both amendments were reviewed and approved by the sponsor and the National Research Ethics Service Committee. Protocol amendments are disseminated to relevant parties by SITU.

\section{Author affiliations}

${ }^{1}$ Academic Urology Unit, University of Sheffield, Sheffield, UK

${ }^{2}$ Division of Surgery and Interventional Science, University College London, London, UK

${ }^{3}$ Department of Statistical Science, University College London, London, UK ${ }^{4}$ Surgical and Interventional Trials Unit (SITU), Division of Surgery and Interventional Science, University College London, London, UK

${ }^{5}$ Department of Urology, Guy's and St Thomas' Hospital, London, UK

${ }^{6}$ Health Economics and Decision Science, NIHR Research Design Service Yorkshire and the Humber, University of Sheffield, Sheffield, UK

${ }^{7}$ Department of Urology, Royal Devon and Exeter NHS Trust, Exeter, UK

${ }^{8}$ Department of Urology, North Bristol NHS Trust, Bristol, UK

Acknowledgements We gratefully thank the participants, principal investigators, research nurses, MDT coordinators, data managers and other site staff who have been responsible for setting up, recruiting participants and collecting the data for the trial. We are grateful for the trial oversight provided by the sponsor, the DMC and the members of the TSC. We thank the iROC steering and data committee members: the iROC Independent Trial Steering Committee (TSC) members are Sam McClinton (Chair), Aberdeen; Grant Stewart, Cambridge; George Thalmann, Switzerland; Alex Mottrie, Belgium; Louise de Winter, London; Frances Christensen, London; and Martin Gibbs, London. The iROC Independent Data Monitoring Committee (DMC) are Roger Kockleburgh (Chair), Leicester; Richard Sylvester, Belgium; Henk van der Poel, The Netherlands.

Contributors Conception and design of the iROC trial: JWFC, JDK, PK, GA, NRW and CB-G. Protocol/patient information sheet: all authors. Writing of manuscript: JWFC, JDK, CB-G, PK, JM, ER, AK, MSK, SD, AF, GA and NRW. All authors have read and approved the final manuscript. The trial will comply with the authorship criteria recommended by the International Committee of Medical Journal Editors.

Funding The trial was funded by The Urology Foundation and Champniss Charitable Trust. JK is funded by the Biomedical Research Centre and Pramit Khetrapal is funded by The Urology Foundation.

Disclaimer The funder had no role in the design, analysis or collection of the data; in writing the manuscript; or in the decision to submit the manuscript for publication.

Competing interests Within IROC, robotic consumables are provider without cost from Intuitive Surgical. JMcG has received educational funding from Intuitive Surgical.

Patient consent Not required.

Ethics approval The study has ethical approval from Newcastle \& North Tyneside Research Ethics Committee (REC reference 16/NE/0418).

Provenance and peer review Not commissioned; externally peer reviewed.

Open access This is an open access article distributed in accordance with the Creative Commons Attribution Non Commercial (CC BY-NC 4.0) license, which permits others to distribute, remix, adapt, build upon this work non-commercially, and license their derivative works on different terms, provided the original work is properly cited, appropriate credit is given, 
any changes made indicated, and the use is non-commercial. See: http:// creativecommons.org/licenses/by-nc/4.0/.

\section{REFERENCES}

1. Antoni S, Ferlay J, Soerjomataram I, et al. Bladder cancer incidence and mortality: a global overview and recent trends. Eur Urol 2017;71:96-108.

2. Svatek RS, Hollenbeck BK, Holmäng S, et al. The economics of bladder cancer: costs and considerations of caring for this disease. Eur Urol 2014;66:253-62.

3. Noon AP, Albertsen PC, Thomas F, et al. Competing mortality in patients diagnosed with bladder cancer: evidence of undertreatment in the elderly and female patients. Br J Cancer 2013;108:1534-40.

4. Babjuk M, Böhle A, Burger M, et al. EAU Guidelines on Non-Muscleinvasive Urothelial Carcinoma of the Bladder: Update 2016. Eur Urol 2017;71:447-61.

5. Shabsigh A, Korets R, Vora KC, et al. Defining early morbidity of radical cystectomy for patients with bladder cancer using a standardized reporting methodology. Eur Urol 2009;55:164-74.

6. Tan WS, Lamb BW, Tan MY, et al. In-depth Critical Analysis of Complications Following Robot-assisted Radical Cystectomy with Intracorporeal Urinary Diversion. Eur Urol Focus 2017;3:30062-1.

7. Eylert MF, Hounsome LS, Persad RA, et al. Falling bladder cancer incidence from 1990 to 2009 is not producing universal mortality improvements. J Clin Urol 2014;7:90-8.

8. Pang KH, Groves R, Venugopal S, et al. Prospective Implementation of Enhanced Recovery After Surgery Protocols to Radical Cystectomy. Eur Urol 2018;73:363-71.

9. Patel HR, Cerantola Y, Valerio M, et al. Enhanced recovery after surgery: are we ready, and can we afford not to implement these pathways for patients undergoing radical cystectomy? Eur Urol 2014:65:263-6.

10. Basse L, Jakobsen DH, Bardram L, et al. Functional recovery after open versus laparoscopic colonic resection: a randomized, blinded study. Ann Surg 2005;241:416-23.

11. Tan WS, Sridhar A, Ellis G, et al. Analysis of open and intracorporeal robotic assisted radical cystectomy shows no significant difference in recurrence patterns and oncological outcomes. Urol Oncol 2016;34:257.e1-257.e9.

12. Yuh $B$, Wilson $T$, Bochner $B$, et al. Systematic review and cumulative analysis of oncologic and functional outcomes after robot-assisted radical cystectomy. Eur Urol 2015;67:402-22.

13. Wilson TG, Guru K, Rosen RC, et al. Best practices in robot-assisted radical cystectomy and urinary reconstruction: recommendations of the Pasadena Consensus Panel. Eur Urol 2015;67:363-75.

14. Hussein AA, Saar M, May PR, et al. Early Oncologic Failure after Robot-Assisted Radical Cystectomy: Results from the International Robotic Cystectomy Consortium. J Urol 2017;197:1427-36.

15. Bochner BH, Dalbagni G, Sjoberg DD, et al. Comparing Open Radical Cystectomy and Robot-assisted Laparoscopic Radical Cystectomy: A Randomized Clinical Trial. Eur Urol 2015;67:1042-50.

16. Khan MS, Gan C, Ahmed K, et al. A Single-centre Early Phase Randomised Controlled Three-arm Trial of Open, Robotic, and Laparoscopic Radical Cystectomy (CORAL). Eur Urol 2016;69:613-21.

17. Smith ND, Castle EP, Gonzalgo ML, et al. The RAZOR (randomized open vs robotic cystectomy) trial: study design and trial update. BJU Int 2015;115:198-205.

18. Nix J, Smith A, Kurpad R et al. Prospective randomized controlled trial of robotic versus open radical cystectomy for bladder cancer: perioperative and pathologic results. Eur Urol 2010;57:196-201.

19. Desai MM, Gill IS. "The devil is in the details": randomized trial of robotic versus open radical cystectomy. Eur Urol 2015;67:1053-5.

20. Stimson CJ, Chang SS, Barocas DA, et al. Early and late perioperative outcomes following radical cystectomy: 90-day readmissions, morbidity and mortality in a contemporary series. $J$ Urol 2010;184:1296-300.

21. Leow JJ, Cole AP, Seisen T, et al. Variations in the Costs of Radical Cystectomy for Bladder Cancer in the USA. Eur Urol 2017;S03022838:30640-1.

22. Kuon E, Dahm JB, Schmitt M, et al. Short communication: time of day influences patient radiation exposure from percutaneous cardiac interventions. Br J Radiol 2003;76:189-91.
23. Butler KA, Kapetanakis VE, Smith BE, et al. Surgeon fatigue and postural stability: is robotic better than laparoscopic surgery? $J$ Laparoendosc Adv Surg Tech A 2013;23:343-6.

24. Wilson MR, Poolton JM, Malhotra N, et al. Development and validation of a surgical workload measure: the surgery task load index (SURG-TLX). World J Surg 2011;35:1961-9.

25. Moore LJ, Wilson MR, Waine E, et al. Robotically assisted laparoscopy benefits surgical performance under stress. J Robot Surg 2015;9:277-84

26. Moore LJ, Wilson MR, Waine E, et al. Robotic technology results in faster and more robust surgical skill acquisition than traditional laparoscopy. J Robot Surg 2015;9:67-73.

27. Ariti CA, Cleland JG, Pocock SJ, et al. Days alive and out of hospital and the patient journey in patients with heart failure: Insights from the candesartan in heart failure: assessment of reduction in mortality and morbidity (CHARM) program. Am Heart J 2011;162:900-6.

28. Herdman M, Gudex C, Lloyd A, et al. Development and preliminary testing of the new five-level version of EQ-5D (EQ-5D-5L). Qual Life Res 2011;20:1727-36.

29. Ustün TB, Chatterji S, Kostanjsek N, et al. Developing the World Health Organization Disability Assessment Schedule 2.0. Bull World Health Organ 2010;88:815-23.

30. Månsson A, Davidsson T, Hunt S, et al. The quality of life in men after radical cystectomy with a continent cutaneous diversion or orthotopic bladder substitution: is there a difference? BJU Int 2002:90:386-90.

31. EORTC. Bladder Cancer: EORTC QLQ-NMIBC24, EORTC QLQBLM30: Belgium. http://groups.eortc.be/qol/bladder-cancer-eortcqlq-nmibc24-eortc-qlq-blm302017.

32. Jones CJ, Rikli RE, Beam WC. A 30-s chair-stand test as a measure of lower body strength in community-residing older adults. Res $Q$ Exerc Sport 1999;70:113-9.

33. Bandinelli S, Milaneschi Y, Ferrucci L. Chair stands test and survival in the older population. J Am Geriatr Soc 2009;57:2172-3.

34. Moore LJ, Wilson MR, McGrath JS, et al. Surgeons' display reduced mental effort and workload while performing robotically assisted surgical tasks, when compared to conventional laparoscopy. Surg Endosc 2015;29:2553-60.

35. Elliott D, Husbands S, Hamdy FC, et al. Understanding and Improving Recruitment to Randomised Controlled Trials: Qualitative Research Approaches. Eur Urol 2017;72:789-98.

36. Mata DA, Groshen S, Von Rundstedt FC, et al. Variability in surgical quality in a phase III clinical trial of radical cystectomy in patients with organ-confined, node-negative urothelial carcinoma of the bladder. J Surg Oncol 2015;111:923-8.

37. Tan WS, Sridhar A, Goldstraw M, et al. Robot-assisted intracorporeal pyramid neobladder. BJU Int 2015;116:771-9.

38. Evenson KR, Goto MM, Furberg RD. Systematic review of the validity and reliability of consumer-wearable activity trackers. Int J Behav Nutr Phys Act 2015;12:159.

39. Dindo D, Demartines N, Clavien PA. Classification of surgical complications: a new proposal with evaluation in a cohort of 6336 patients and results of a survey. Ann Surg 2004;240:205-13.

40. Clavien PA, Barkun J, de Oliveira ML, et al. The Clavien-Dindo classification of surgical complications: five-year experience. Ann Surg 2009;250:187-96.

41. U.S Department of Health and Human Services, National Institute of Health. Common Terminology Criteria for Adverse Events (CTCAE) Version 4.0. 2009 http://evs.nci.nih.gov/ftp1/CTCAE/CTCAE_4.03_ 2010-06-14_QuickReference_5x7.pdf.

42. Thompson JE, Egger S, Böhm M, et al. Superior quality of life and improved surgical margins are achievable with robotic radical prostatectomy after a long learning curve: a prospective singlesurgeon study of 1552 consecutive cases. Eur Urol 2014;65:521-31.

43. Yaxley JW, Coughlin GD, Chambers SK, et al. Robot-assisted laparoscopic prostatectomy versus open radical retropubic prostatectomy: early outcomes from a randomised controlled phase 3 study. Lancet 2016;388:1057-66

44. Haglind E, Carlsson S, Stranne J, et al. Urinary Incontinence and Erectile Dysfunction After Robotic Versus Open Radica Prostatectomy: A Prospective, Controlled, Nonrandomised Trial. Eur Urol 2015;68:216-25.

45. Herlemann A, Cowan JE, Carroll PR, et al. Community-based Outcomes of Open versus Robot-assisted Radical Prostatectomy. Eur Urol 2018;73. 
Correction: Robot-assisted radical cystectomy with intracorporeal urinary diversion versus open radical cystectomy (iROC): protocol for a randomised controlled trial with internal feasibility study

Catto JWF, Khetrapal P, Ambler G, et al. Robot-assisted radical cystectomy with intracorporeal urinary diversion versus open radical cystectomy (iROC): protocol for a randomised controlled trial with internal feasibility study. BMJ Open 2018;8:e20500. doi:10.1136/bmjopen-2017-020500.

This article was previously published with an error.

The funder's name is the Champniss Foundation, and not the Champniss Charitable Trust.

Open access This is an open access article distributed in accordance with the Creative Commons Attribution Non Commercial (CC BY-NC 4.0) license, which permits others to distribute, remix, adapt, build upon this work non-commercially, and license their derivative works on different terms, provided the original work is properly cited, appropriate credit is given, any changes made indicated, and the use is non-commercial. See: http://creativecommons.org/licenses/by-nc/4.0/

(C) Author(s) (or their employer(s)) 2019. Re-use permitted under CC BY-NC. No commercial re-use. See rights and permissions. Published by BMJ.

BMJ Open 2019;9:e020500corr1. doi:10.1136/bmjopen-2017-020500corr1 